Casos Clínicos

Arch. Esp. Urol. 2009; 62 (8): 667-671

\title{
LINFOCELE GIGANTE TRAS TRASPLANTE RENAL. A PROPÓSITO DE UN CASO Y REVISIÓN DE LA LITERATURA
}

Daniel López García, José Manuel Janeiro Pais, Juan González Dacal, Antón Zarraonandía Andraca, Pastor Casas Agudo, Sara Martínez Breijo, Luis Álvarez Castelo, Manuel Ruibal Moldes, Venancio Chantada Abal y Marcelino González Martín.

Servicio de Urología. Hospital Juan Canalejo. La Coruña. España.

Resumen.- OBJETIVOS: Comunicar el caso y la iconografía de un linfocele postrasplante renal y revisar la literatura sobre diagnóstico y tratamiento de esta complicación quirúrgica

MÉTODOS: Mujer de 69 años sometida a trasplante renal, que se presenta con edema en miembro inferior derecho y deterioro de función renal. Se demostró con ecografía y TAC una colección líquida, compatible con linfocele tras estudio bioquímico del líquido obtenido por punción percutánea.

Daniel Lopez García

Paseo Marítimo, 9, 5D.

15002 La Coruña (España).

delnilopez@hotmail.com

Centro de trabajo:

Servicio de Urología, planta 11

Hospital Juan Canalejo

Xubias de Arriba s/n

15006 La Coruña (España).

Trabajo recibido: 13 de marzo 2009. 
RESULTADOS: Se somete a la paciente a una marsupialización del linfocele por vía laparoscópica, con buenos resultados quirúrgicos.

CONCLUSIONES: El linfocele es una entidad frecuente tras el trasplante renal, precisando tratamiento según las manifestaciones clínicas. Existen fundamentalmente dos alternativas terapéuticas, dependiendo del tamaño: escleroterapia y marsupialización quirúgica. Salvo casos muy seleccionados, el abordaje laparoscópico se considera actualmente, por su seguridad y eficacia, de primera elección cuando se indica tratamiento quirúrgico.

Palabras clave: Linfocele. Trasplante renal.

Summary.- OBJECTIVES: To report the case and iconography of a lymphocele after renal transplantation and to review the literature about the diagnosis and the treatment of this surgical complication.

METHODS: 69 year-old woman status post renal transplantation who presents right lower extremity edema and worsening renal function. A liquid collection was demonstrated by ultrasound and computerized tomography, compatible with lymphocele after biochemical study of the liquid obtained by percutaneous puncture.

RESULTS: The patient underwent laparoscopic intraperitoneal drainage of the lymphocele, with good surgical out come.

CONCLUSION: Lymphocele is a common pathology after a renal transplantation which needs to be treated depending on its clinical manifestations. There mainly are two therapeutic alternatives depending on the size of the lymphocele: sclerotherapy and surgical intraperitoneal drainage. Apart from very selected cases, laparoscopic approach is currently considered, because of its security and effectiveness, the first choice when a surgical treatment is prescribed.

Keywords: Lymphocele. Renal transplantation.

\section{INTRODUCCIÓN}

La presencia de colecciones líquidas en el postoperatorio y en la evolución a medio-largo plazo de un trasplante renal es un hecho frecuente, demostrándose en algunas series hasta en el $50 \%$ de los casos $(1,2)$, la mayoría asintomáticos.

En las ocasiones en las que existe una repercusión general, vascular o sobre la vía urinaria secundaria a dicha colección, está indicado filiar su naturaleza.
El estudio de la colección se orienta a distinguir entre urinoma, absceso, linfocele y hematoma, mediante la cronología, las pruebas de imagen (ECO, TAC) y el análisis del líquido.

\section{CASO CLÍNICO}

Presentamos el caso de una paciente de 69 años con antecedentes de hipertensión arterial e hipercolesterolemia, afecta de insuficiencia renal crónica terminal de etiología no filiada, con anemia nefrogénica tratada con factores estimulantes de la eritropoyesis e hiperparatiroidismo secundario. La diuresis residual se situaba en torno a $200 \mathrm{ml}$. al día.

En el año 2004 inicia tratamiento sustitutivo de la función renal mediante hemodiálisis, entrando posteriormente en lista de espera de trasplante renal.

Recibe en enero de 2008 un trasplante renal de donante cadáver en fosa ilíaca derecha. El postoperatorio es correcto salvo dos complicaciones médicas: desarrollo de diabetes mellitus que precisa administración de insulina y toxicidad por anticalcineurínicos, la cual supone que inicialmente el injerto no sea funcionante. Es dada de alta en la quinta semana tras la intervención con creatinina de $0,9 \mathrm{mg} / \mathrm{dl}$ y diuresis del orden de 2000 $\mathrm{ml}$. al día, recibiendo triple terapia inmunosupresora con prednisona, tacrolimus y micofenolato mofetil.

Reingresa dos semanas después por un cuadro consistente en importante edema en miembro inferior derecho y deterioro de la función renal, con disminución de la diuresis y elevación de la concentración de creatinina en plasma hasta $2 \mathrm{mg} / \mathrm{dl}$. Mantenía un buen estado general, estando ausentes la fiebre y las manifestaciones hemodinámicas.

La ECO demuestra una gran colección peri-injerto, estudiada posteriormente con TAC, que se extiende desde un nivel algo más craneal que la bifurcación aór-

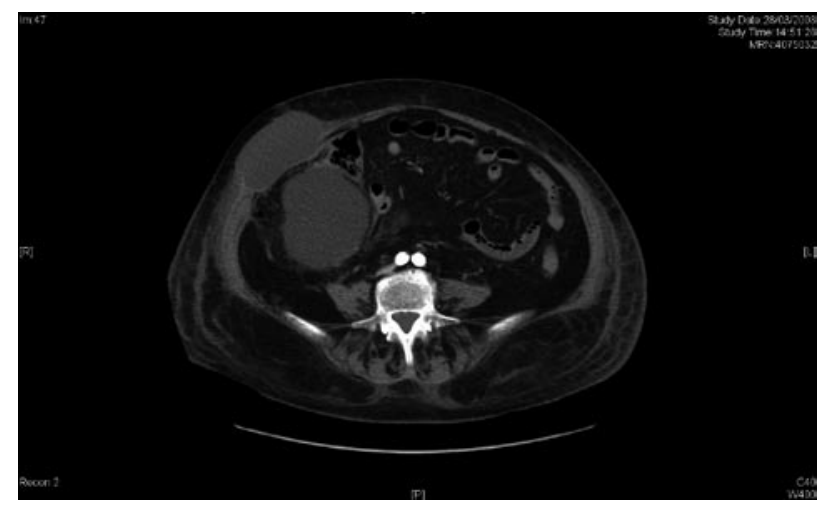

FIGURA 1. Nivel craneal de la colección. 


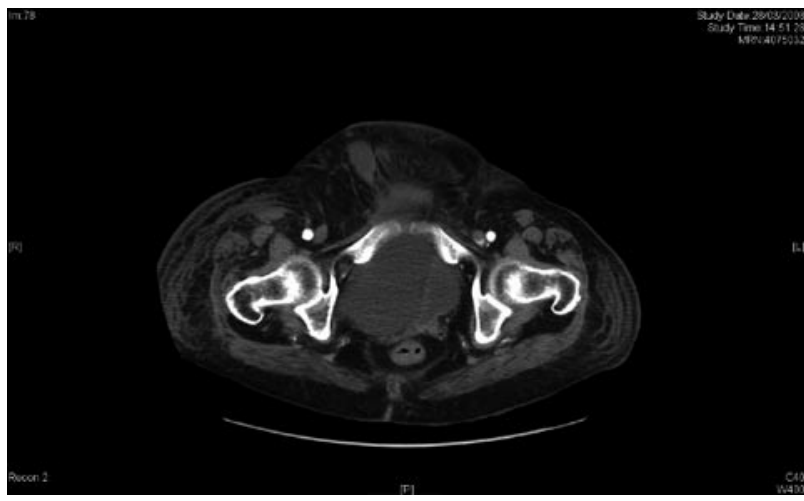

FIGURA 2. Nivel caudal de la colección.

tica (Figura 1) hasta el recto (Figura 2), produciendo compresión de los vasos ilíacos y deplazamiento de la vejiga (Figura 3).

La punción percutánea y colocación de pig-tail para drenar la colección se sigue de una gran mejoría de los síntomas y el análisis bioquímico del líquido obtenido (creatinina $1,67 \mathrm{mg} / \mathrm{dl}, \mathrm{Na} 146 \mathrm{mEq} / \mathrm{l}, \mathrm{K} \mathrm{4,3}$ $\mathrm{mEq} / \mathrm{ll}$ confirma la sospecha clínica de linfocele.

Se indica tratamiento quirúrgico, llevando a cabo, una vez descartada la infección, marsupialización del linfocele a cavidad peritoneal por vía laparoscópica. La paciente recibe el alta quirúrgica el tercer día postoperatorio, con función renal normal y asintomática.

En la TAC de control a los 14 días se comprueba la resolución del linfocele (Figura 4).

\section{DISCUSIÓN}

El linfocele es una colección de linfa ubicada en el lecho quirúrgico. Hume la describe por primera vez en relación con un trasplante renal en 1968 (3), de-

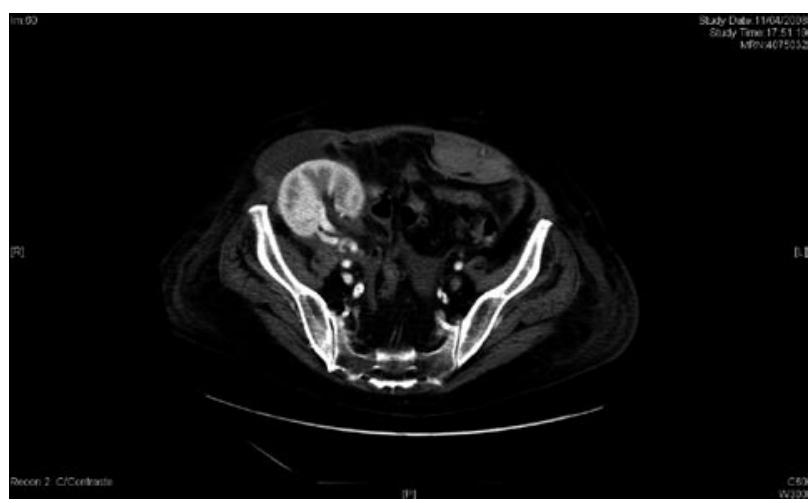

FIGURA 4. TAC de control, colección resuelta.

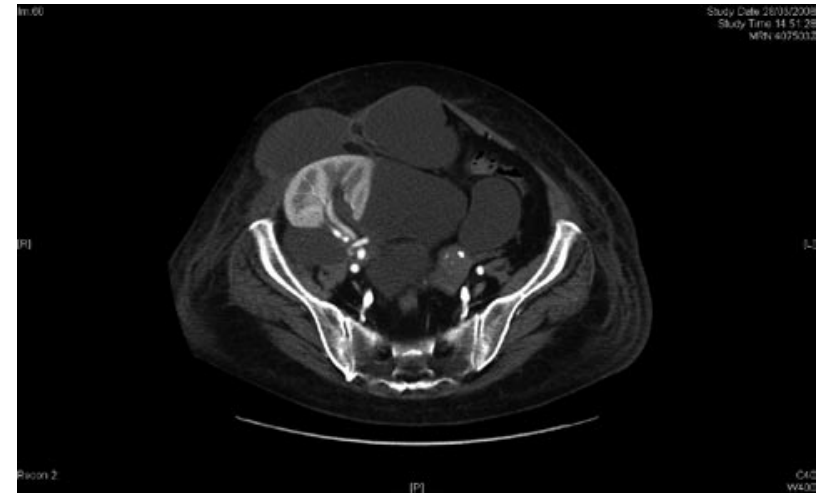

FIGURA 3. Colección abarcando vasos ilíacos, uréter del injerto y desplazando la vejiga.

mostrándose desde entonces con frecuencia elevada, hasta en un $18 \%$ de los casos (4), aunque sólo precisa tratamiento en torno a un $5 \%$ (5).

En su etiología se ven envueltos factores médicos y quirúrgicos. De entre los médicos se destaca clásicamente el rechazo agudo (6), que tiene una importancia cada vez menor, inversamente al desarrollo de la medicación inmunosupresora; también se ha relacionado la terapia inmunosupresora basada en inhibidores de mTOR con una mayor incidencia de linfoceles (7) y complicaciones de la herida quirúrgica $(8,9,10)$. Los factores quirúrgicos parecen ser los más determinantes, y se señala la lesión de los vasos linfáticos del injerto sobre todo (11), aunque también de los perivasculares de la región de las anastomosis, como la causa fundamental; también se ha implicado la decapsulación del órgano, aunque en un segundo plano.

Se llega al diagnóstico siguiendo una sospecha clínica. Los síntomas y signos del linfocele clínicamente significativo son los derivados de la compresión de estructuras vecinas: tumoración en fosa ilíaca o hipogastrio, edema en el miembro inferior ipsilateral, obstrucción de la vía urinaria alta o baja, estreñimiento, hipertensión, trombosis venosa. Debe integrarse la semiología según su tiempo de aparición, teniendo en cuenta que el linfocele suele presentarse a partir del primer mes del trasplante (6). La ecografía y la TAC son las pruebas de imagen más útiles, y rara vez se utilizan RNM o radioisótopos. Se confirma el diagnóstico con el análisis bioquímico del líquido, que tendrá una composición similar a la del suero.

Cuando un linfocele debe ser tratado, es necesario valorar las alternativas con las que se cuenta:

- Punción-aspiración percutánea: poco utilizada por los pobres resultados y el alto riesgo de recidiva e infección $(6,12)$.

- Drenaje quirúrgico externo: más eficaz que la aspiración, presenta también el inconveniente del riesgo de 
infección y se añade el tiempo prolongado de tratamiento (6). Tampoco suele indicarse esta maniobra.

- Marsupialización: es una intervención quirúrgica consistente en abrir una ventana que comunique el linfocele con la cavidad peritoneal para que aquí se reabsorba la linfa. Tiene excelentes resultados y baja morbilidad. Puede llevarse a cabo por vía abierta o laparoscópica, actualmente de primera elección (12-14) salvo excepciones y asociarse o no a omentoplastia para incrementar la capacidad de reabsorción y disminuir el riesgo de recidiva (6). La marsupialización exige descartar previamente infección del linfocele.

- Escleroterapia: consta de dos pasos: punción de la colección e introducción de sustancias a la vez antibacterianas y esclerosantes en la cavidad hasta colapsarla, habiéndose utilizado tetraciclinas, etanol, ampicilina y muchas otras. En nuestro Servicio hemos sido pioneros en la utilización de povidona yodada, tratamiento que iniciamos en 1982 (15), obteniendo desde entonces muy buenos resultados.

De las cuatro modalidades terapéuticas, la marsupialización y la escleroterapia son las más usadas, sentando la indicación de una u otra casi siempre el tamaño (16), y en ocasiones la localización (5).

\section{CONCLUSIONES}

Las colecciones líquidas periinjerto renal son una entidad muy frecuente (2), aunque en pocas ocasiones precisan tratamiento, el cual se indica según la repercusión clínica.

El estudio diagnóstico está encaminado a determinar la naturaleza de la colección de entre las cuatro fundamentales: absceso, hematoma, urinoma y linfocele. El protocolo habitual en nuestro Centro tiene como prueba clave en el diagnóstico los análisis microbiológico y bioquímico del líquido obtenido mediante punción percutánea de la colección y se apoya en pruebas de imagen, casi siempre ecografía y TAC.

Para tratar el linfocele existen varias opciones, decantándose la decisión hacia escleroterapia o hacia marsupialización según las características del caso y el tamaño de la colección. La marsupialización laparoscópica es un método seguro, eficaz y relativamente sencillo, y es considerado de primera elección frente a la vía abierta cuando se indica tratamiento quirúrgico (12-14), salvo indicaciones concretas de cirugía abierta, como los casos en los que existe una complicación de la herida quirúrgica o linfoceles pequeños en íntima relación con estructuras vitales para el injerto renal (14).

El caso clínico que se presenta fue tratado mediante marsupialización laparoscópica, atendiendo al tama- ño de la colección; los resultados quirúrgicos fueron buenos.

\section{BIBLIOGRAFÍA y LECTURAS RECOMENDADAS ( ${ }^{*}$ lectura de interés $y^{* *}$ lectura fundamental)}

1. Abid Irshad, MD, Susan Ackerman, MD, David Sosnouski, MD, Munazza Anis, MD, Kenneth Chavin, MD, and Prabhaker Baliga, MD. A Review of Sonographic Evaluation of Renal Transplant Complications. Curr Probl Diagn Radiol 2008;37:67-79.

**2. Secin F P, Rovegno A R, Marrugat R E J, Virasoro R, Lautersztein G A, Montenegro L C, Fernández H. Valor de la ultrasonografía en escala de grises en el diagnóstico precoz de las complicaciones urológicas del trasplante renal. Arch Esp Urol, 2002; 55 (4):395-404.

3. Hume DM: Kidney transplantation in Rapapaport FT, Dansen J (eds): Human Transplantation. New York. Grune Stratton Inc 1968. Pag 110 - 150.

4. Samhan M, Al-Mousawi M. Lymphocele Following Renal Transplantation. Saudi J Kidney Dis Transpl 2006;17:34-7.

**5. Burgos Revilla F J, Marcen Letosa R, Pascual Santos J, López Fando L. Utilidad de la ecografía y el eco-doppler en el trasplante renal. Arch Esp Urol, 2006; 59 (4):343-352.

*6. González Martín M, Gómez Veiga F, Álvarez Castelo L. Colecciones periinjerto en el trasplante renal. En: González Martín, M., García Buitrón, J.M. Trasplante renal. Madrid: Aula Médica Ediciones; 2000. p. $379-391$.

7. Quicios Dorado C, Burgos Revilla F J, Pascual Santos J, Marcén Letosa R, Gómez García I, García Navas R, De Castro Guerín C. Inmunosupresión y complicaciones quirúrgicas post-trasplante renal. Arch Esp Urol, 2006; 59, 7 :697-705.

8. Knight R J, Villa M, Laskey R, Benavides C, Schoenberg L, Welsh M, Kerman R H, Podder H, Van Buren CT, Katz SM, Kahan BD. Risk factors for impaired wound healing in sirolimus-treated renal transplant recipients. Clin Transplant, 2007; 21(4):460-5.

**9. Derweesh I H, Ismail H R, Goldfarb D A, Araki M, Zhou L, Modlin C, Krishnamurthi V, Flechner SM, Novick A C. Intraoperative placing of drains decreases the incidence of lymphocele and deep vein thrombosis after renal transplantation. BJU Int. 2008; 101(11):1415-9. Epub 2008 Jan 10.

10. Langer R, Tóth A, Máthé Z, Remport A, Járay J, Kahan BD. Lymphocele and kidney transplantation. Orv Hetil. 2007 Aug 5;148(31):1475-80.

**11. Saidi R F, Wertheim J A, Ko D S, Elias N, Martin H, Delmonico F L, Cosimi A B, Kawai T. Impact of donor kidney recovery method on lymphatic complications in kidney transplantation. Transplant Proc. 2008 May;40(4):1054-5. 
12. S. H. Bailey, M. C. Mone, J. M. Holman, E. W. Nelson. Laparoscopic treatment of postrenal transplant lymphoceles. Surg Endosc (2003) 17: 1896-1899

13. Doehn C, Fornara P, Fricke L, Jocham D. Laparoscopic fenestration of posttransplant lymphoceles. Surg Endosc. 2002 Apr;16(4):690-5. Epub 2001 Dec 31.

14. Fuller TF, Kang SM, Hirose R, Feng S, Stock PG, Freise CE. Management of lymphoceles after renal transplantation: laparoscopic versus open drainage. $\mathrm{J}$ Urol. 2003 Jun;169(6):2022-5.

**15. González Martín M, García Buitrón JM, Lancina Martín A, Oliver García J, Barrientos Guzman A. A new method of treating lymphatic complications in renal transplants: intracavitary instillations of povidone-iodide. Actas Urol Esp. 1985 Jul-Aug;9(4):2878.

**16. Król R, Kolonko A, Chudek J, Ziaja J, Pawlicki J, Ma_y A, Kunsdorf-Wnuk A, Cierpka L, Wiecek A. Did volume of lymphocele after kidney transplantation determine the choice of treatment modality? Transplant Proc. 2007 Nov;39(9):2740-3. 